\title{
ESPACIOS ABIERTOS Y YACIMIENTOS RECIENTEMENTE IDENTIFICADOS EN MADRE DE DIOS
}

\author{
Wilber Bolívar Yapura' \\ RuBÉN MAQQUe AZORSA
}

E n el área de madre de Dios, las investigaciones arqueológicas son muy escasas, por lo que el presente artículo busca en los antecedentes arqueológicos y etnoarqueológicos una idea de cómo se da el proceso de poblamiento de esta vasta área, incidiendo en la habilitación de los espacios abiertos, cuyo uso como espacio público estaba relacionado con su entorno. Se trata de buscar conceptos viables de yacimientos arqueológicos y su naturaleza, para aplicar en la descripción de los emplazamientos recientemente ubicados. Finalmente tratar de describir los motivos decorativos desde el punto de vista morfológico.

In Madre de Dios area, archaeological investigations are very scarce, and so the present article looks for the archaeological and etnoarqueogical antecedents an idea the process of poblamiento of this vast area is given of how, impacting in the qualification of the open spaces whose use like public space was related with its environment. It is to look for viable concepts of archaeological locations and their nature, to apply in the description of the recently located locations. Finally to try to describe the ornamental reasons from the morphological point of view.

Universidad Nacional De San Antonio Abad del Cusco - Universidad Andina Cusco.

INC - Madre de Dios 


\section{Preámbulo}

En la Amazonía no se ha realizado mucha investigación arqueológica, situación que cambia gracias a los trabajos realizados por investigadores extranjeros y nacionales, entre los que tenemos a Donald Lathrap, Betty Meggers, C. Evans, Warren De Boer, Thomas Myres y a los peruanos Roger Ravines, Ducio Bonavía, Luís Lumbreras, Rosa Fung, D. Morales, C. Dávila, V. Chang, entre otros que prestan atención a la investigación arqueológica de la Amazonía.

Sin embargo, en Madre de Dios el retraso en este tipo de investigaciones es mucho más notable, por lo que debemos tomar en cuenta una notoria carencia de análisis, de pistas del pasado, carencia que impide situar en el tiempo la antigüedad del poblamiento en la región, debido especialmente a que las condiciones climáticas resultan poco propicias para la conservación de los restos culturales.

Aún sin mencionar a Madre de Dios, resulta evidente que en esta gran área geográfica existieron poblaciones por las mismas épocas. Se puede afirmar con bastante probabilidad que hace 3,000 años había gente "produciendo cultura». Esta Selva Baja de la Amazonía peruana es más homogénea y concuerda con la descripción de la llanura amazónica con sus dos características ecológicas de «tierra firme» o «montaña» y la zona de «várcea» o «zonas inundables», cuyo proceso histórico cultural ha sido estudiado profundamente por Donald Lathrap (1970) y Betty Meggers (1975), quienes las han denominado como culturas de bosque tropical.

Esto se puede percibir a través del estudio etnohistórico, el mismo que recoge evidencias arqueológicas, lingüísticas y la tradición oral. Un caso concreto son los Matsigenka (Machiguengas), que aún viven en Madre de Dios y Cuzco, por lo que podemos inferir que su trayectoria histórica y proceso de adaptación les permitió afrontar al incario y las primeras penetraciones españolas.

\section{Antececedentes Arqueologicos:}

La investigadora S. Aikman $(1980)^{3}$ efectúa prospecciones en el río Karene (Colorado), afluente de la cuenca del río Madre de Dios; identificando los yacimientos arqueológicos de Puerto Luz, Palizada, San José, Chapahal A y B, los mismos que consisten en fragmentos de cerámica sencilla y pintura roja; llegan a registrar un cuenco con ligero bisel interior, de cuerpo cóncavo y base redondeada; ollas con perfil en forma de «S», con cuello mediano evertido, cuerpo globular y base convexa, además de fragmentos de cuerpo carenado. También hay hachas en forma de «T» algo pronunciadas, al parecer de una larga tradición tecnológica en zonas de selva,

Rivas Panduro, Santiago. «Los Asentamientos pre hispánicos de la Cuenca del Rió CachiyacuAmazonía Peruana», 2003: 1-141. 
cuyo análisis si lo relacionamos con otros hallazgos, como vías y estructuras incaicas identificadas en la ceja de la selva del Cusco y Madre de Dios, sugiere la existencia de relaciones entre la selva y la sierra.

R. Ravines (1984) ${ }^{4}$ investiga los yacimientos de Sandoval y Santa María (Tambopata - Madre de Dios), complementando estos en 1994 con el yacimiento de Playa del Águila (Laberinto - Tambopata). Al parecer el material arqueológico procede de un cementerio ubicado en la terraza del río, el mismo que consiste en vasijas de cuerpo compuesto, pedestales altos, con decoración pintada con diseños geométricos en colores rojo sobre blanco, asociados a objetos de oro laminado. Uno de los artefactos cerámicos tiene aplicaciones consistentes en incisiones, en un patrón de volutas, que se asemejan a los motivos decorativos de los Piro actuales, cuyas formas y decoración correlacionan una aparente relación con la tradición Cumancaya del Ucayali Central.

M. Panaifo (1994-1996) ${ }^{5}$ explora en Madre de Dios, identificando evidencias de culturas prehispánicas en los poblados de Mazuco, Choque, Hueypetue, Quincemil, Patria, Shintuya, Marcapata, Inambari, Tambopata y Colorado. Manifiesta la presencia de estilos pre-incas desarrollados y extendidos en el Bajo Urubamba, como Shakimu, Cumancaya y Caimito.

P.M. Vega Centeno (2003) ${ }^{6}$ estudia los petroglifos de Pusharo (rió Sinkibenia, tributario del Palotoa), en la cordillera del Pantiaqolla, emplazado en la región Manu, logrando identificar 310 figuras grabadas, destacando las formas geométricas o abstractas (círculo, línea, punto, espirales, rombos, cruces, cuadrados, zig-zags), y en menor porcentaje figuras zoomorfas y antropomorfas, como rostros antropomorfizados a manera de máscaras, serpientes, ranas, pisadas de animales como el jaguar y aves, círculos concéntricos, espirales simples y dobles contrapunteados, rectángulos, triángulos, de modo que la presencia constante de las mismas formas demuestra que se trata de un mismo conjunto estilístico.

R. Maqque A. $(2005)^{7}$ y W. Bolívar $(2006)^{8}$, en Madre de Dios, Provincia de Tambopata y Puerto Maldonado, identifican yacimientos arqueológicos emplazados

$4 \quad$ Rogger Ravines. «Cerámica Arqueológica del Rió Tambopata, Madre de Dios». En Boletín de Lima. Vol. XV, No 90, Año 15, 1993: 15-20.

5 Mónica Panaifo Teixeira. «Estudios de Impacto Ambiental. Proyecto Sistemático Karene-Madre de Dios-Perú». En: Walsh Envioremental Scientists and Engineers. (1996: 45).

6 Patricia Vega Centeno A. «Algunos Apuntes sobre la Investigación Etno-arqueológica de un sitio con Petroglifos en la Selva Peruana». En: Arqueológicas No 26, del Museo Nacional de Arqueología, Antropología e Historia del Perú. 2003: 59-72.

Ruben Maqqe A. «Identificación de los Sitios Arqueológicos en Madre de Dios». En: estudios Amazónicos: Madre de Dios en la encrucijada, No 3 año II, 2005: 77-88

8 Bolívar, Y. Wilber. «Informe N N 083 - WBY-DRC-C-DCPCI-SDCH-2006» Sobre trabajos para delimitacion de sitios arqueológicos de El Triunfo y Pampa Hermosa en al Provincia de TambopataMaldonado- Madre de Dios. Presentado a la Dirección Regional del INC-Cusco, 2006. 
sobre terrazas, entre las que destacan El Triunfo y Santa María, lugares en los que se registran fragmentos de cerámica consistentes en vasijas abiertas (platos, cuencos y tazones), con predominio de cerámica corrugada e incisa de trazos geométricos.

\section{Análisis de los antecedentes etno-arqueologicos}

Los estudios nos indican la existencia de dos troncos lingüísticos para la Amazonía: Tupi Guarani y Arawak o Arahuaco. Este último representa la mayor dispersión geográfica en la América Tropical.

Los grupos Arawac, en territorio peruano se encontraban a lo largo de las estribaciones andinas (orientales peruano y boliviano); distinguiéndose dos núcleos: el primero, localizado en el piedemonte andino peruano, a lo largo de los ríos que forman el Ucayali. En la zona de los pueblos de habla Arahuaca (Matsiguenga, Ashaninka, Asheninka, Nomatsiguenga, Yine-Piro, y Yanesha). Y el segundo, se encontraba ubicado en el oriente boliviano, a lo largo de los ríos Beni y Mamore, concluyentes del Madeira, que desemboca en el Amazonas ${ }^{9}$.

Latrhap $(1970)^{10}$, utilizando información histórica, lingüística, geográfica, demográfica y arqueológica, plantea el modelo demográfico de doblamiento amazónico, llamada también zonificacion ecológica de la Amazonía, según la cual los dialectos de las poblaciones más antiguas de la Amazonía pertenecen al tronco lingüístico Arawac, que inicialmente ocupaban la «varzea» o zonas ribereñas, donde podían contar con los mejores recursos de pesca, caza acuática y agricultura intensiva, capaz de producir excedentes suficientes para lograr un sedentarismo de grandes poblaciones, con desarrollo sociocultural complejo. Estos factores ocasionaron el aumento poblacional, ejerciendo presión en el territorio de la várzea, provocando expulsiones de poblaciones excedentes hacia las zonas de la montaña y contrafuertes andinos, y manteniendo permanentes conflictos de recursos.

Luego sugiere el centro de origen de las poblaciones Proto-arawac originales, que sería el medio Amazonas, en las cercanías de la desembocadura del río Negro, área en donde la expulsión de excedentes de población habría ocurrido a partir de los 3000 a.C. Uno de los grupos expulsados llegaría al Ucayali Central hace aproximadamente 2000 a.C. Estos datos se fundamentan en base a sus excavaciones arqueológicas en la zona, correlacionadas con el estilo cerámico Tutishcaindio Temprano.

\footnotetext{
$9 \quad$ Lucia Medina de la C. «Informe del Proyecto Arqueológico de Emergencia en Niateni Shianiato, Sitio 6, Lote 56, bajo Urubamba, Cusco». Pluspetrol Perú Corporation S. A., 2007.

10 Donald Lathrap. «The Upper Amazon», 1970: 74.
} 
M. Heckenberger (2002) ${ }^{11}$ cuestiona esta hipótesis, ya que no existen evidencias arqueológicas que indique un crecimiento poblacional significativo hacia los años 3000 a.C., momento en que las diversas familias lingüísticas arahuacas empiezan a separarse, indicando que la diáspora arahuaca debe buscarse en la micro dinámica sociopolítica de los asentamientos locales.

Meggers (1983) plantea el modelo biogeográfico del doblamiento amazónico, tomando en cuenta la teoría de que la Amazonía habría sufrido fuertes procesos de desecación, aproximadamente entre los años 5000 a 2500 A.P., creando los «refugios de bosques» o «centros de dispersión» de plantas y animales que plantearon los biólogos. Tal biodiversidad, ocasionaría la diversificación en los grupos humanos, su cultura y lenguas, refugiados en los bosques relixtos de la foresta amazónica, siendo uno de estos la región oriental del Perú (Prance; 1973) ${ }^{12}$.

Para probar sus hipótesis muestra mapas etno-lingüísticos con la diversificación de los grupos étnicos de la Amazonía como los Arawac y TupiGuarani. Estos episodios climáticos, serían los responsables de los mecanismos de doblamiento por migraciones, ocasionando la baja densidad poblacional e itinerante, que limitaron su desarrollo cultural.

Meggers y Evans (1982), diferenciaron la dispersión de la familia Maipure del tronco Arahuac y su correlación con tradiciones cerámicas está aún por determinarse. Las informaciones disponibles sugieren una heterogeneidad en cuanto a materiales cerámicos, donde la vajilla decorada con líneas incisas anchas y zonas del rayado fino, hallada en el Bajo Amazonas y en la Isla Marajó, podría estar relacionadas a ésta.

«La cerámica, achurada en esta zona parece derivarse del área andina, unido con la existencia de grupos residuales Arawak en los altiplanos peruanos y bolivianos, permite deducir la posibilidad de una antigua conexión» ${ }^{13}$.

Los estudios acerca de la clasificación del tronco Tupi-Guarani (Rodríguez, 1958), hacen mención de cinco familias ubicadas al Sur-oeste de la hoya Amazónica, cuyo lugar de origen sería la planicie amazónica al este del río Madeira, en los límites del Estado de Amazona (Brasil) y Bolivia. La antigüedad de la separación (cerca de 5000 a.C.), sugiere que la diferenciación comenzó cuando los hablantes eran aún pre-agricultores y no se daba producción cerámica (Meggers y Evans, 1982: 21).

11 Michael Heckenberger. Rethinking te Arawakan diaspora: Hierarchy, regionality, and the Amazonían formative» (2001:101). En: Santos y Barclay.» Guía Etnográfica de la Alta Amazonía». 2004.

12 B. Meggers; C. Evans. «Aplicación del Modelo Biológico de Diversificación a las Distribuciones Culturales en las Tierras tropicales Bajas de Sudamérica». En Amazonía Peruana. Vol. IV, $\mathrm{N}^{\circ}$ 8, 1993: 9

13 B. Meggers; C. Evans. «La reconstrucción de la Pre-historia Amazónica: Algunas Consideraciones Teoricas». Amazonía Peruana. Vol IV, No 7, 1982: 24 
Las relaciones de evidencias en cerámica distribuidas en territorios de habla Tupi-Guarani permitieron una recontracción regional que comprende desde los Estados de Río Grande del Norte y Sur (Brochado: 1969).

Meggers y Evans (1982), mencionan que los restos arqueológicos designados como Tupi-Guarani, consisten en artefactos fragmentados de cerámica y piedra con características que son universales y con variaciones locales en forma y técnicas decorativas. Los rasgos diagnóstico son: «... la decoración pintada de rojo y/o negro sobre superficie recubierta de blanco y la corrugada, angulada, punteada y engobe rojo están frecuentemente asociados...»

Las investigaciones etnolingüísticas de ambos troncos Arawak y Tupi Guarani, sugieren que la dispersión fue simultánea. Noble (1965), utilizando métodos léxicoestadísticos, fecha esa separación entre los 2800 y 2500 años atrás, cuyas cercanías de los centros de origen sugieren que ambos troncos derivaron de un ancestro común (Greenberg, 1960; Noble, 1965. 9).

Existen en el territorio amazónico dos movimientos migratorios: Tupi Guarani, proveniente de la parte Sur central de la llanura amazónica, hincadas desde el corredor de las selvas del Beni (Bolivia), para proseguir por los cauces llegando a la cuenca de Madre de Dios, Manu, el Alto Urubamba y el Ucayali, que es el punto donde se da su mayor expansión en la llanura amazónica. La otra corriente migratoria, provenía del Norte de la llanura Amazónica (Arahuac) y el otro desde el Sur, para ocupar la parte central de la cuenca. Ocurridas, debido a los cambios climáticos, se produjeron tres periodos de sequías, dando lugar a movimientos migratorios y los consecuentes conflictos inter-étnicos, por la escasez de recursos de subsistencia, adaptándose a las nuevas condiciones de refugios forestales, generándose la delimitación territorial en las principales cuencas de la llanura amazónica.

B. Meggers $(1977)^{14}$, realiza criticas en torno a la confiabilidad de los datos etno históricos, para deducir la densidad poblacional y nivel de complejidad cultural alcanzado por los habitantes de la Amazonía: de la llanura aluvial (várzea), y las tierras bajas adyacentes (tierra firme) para sobrevivir, determina la necesidad de depender de los recursos de la tierra firme para sobrevivir, determina además la necesidad sostenible de las poblaciones de la várzea; donde las poblaciones pasadas tuvieron una tecnología mas desarrollada que las actuales.

14 B. Meggers; C. «la Amazonía en vísperas del contacto europeo: perspectivas etnohistóricas, ecológicas y antropológicas» En: Arqueología, Antropología e Historia en los Andes. 1997: 93-113. 


\section{Espacios abiertos y su entorno.}

Estos espacios también pueden ser considerados como el arquetipo del espacio público, en la que se definía la mayoría de las prácticas sociales, es decir en ella se producían simultáneamente las condiciones de intervención en la propia sociedad o como participes en una empresa en la que actuaban grupos y clases con objetivos propios y diferenciados, esto desde el punto de vista del comportamiento humano

Desde la perspectiva de la infraestructura seria determinar la relación existente entre uso del espacio abierto (espacio ganado a la selva a través del roce) y la naturaleza del poder social, el mismo que se ha convertido en un tema de investigación principal en los discursos antropológicos y arqueológicos actuales. Por ejemplo en los asentamientos de gran escala y suficientemente peculiares, el espacio «varía entre privado y público en diseño y uso, y es configurado en numerosos ámbitos por los habitantes» (Smith 2003b:19) los cuales circunscriben zonas con viviendas, áreas de producción como talleres, edificios públicos y/o comunales, etc.

La condición «espacio público» incluye, plazas, edificios y otras áreas a las que puede acceder gran cantidad de individuos, estos espacios públicos se pueden diferenciar por su grado y confección y, lo más importante en este contexto, su forma y orientación pueden ser utilizados para imponer el acceso y uso de áreas.

Entonces los espacios públicos son áreas de encuentros significativos para todos los sectores de la sociedad, constituyen el espacio central en la que las relaciones sociales, económicas y políticas, que se reflejan originalmente en su construcción se reproducen, negocian y, a menudo, se desafían.

Por lo que aparecen nuevos conceptos, como la relación hombre-medio, los estudios macro y microambientales, la teoría del lugar central de Christaller y las relaciones entre el ecosistema y la esfera socio-cultural.

Lo más reciente es pues la aplicación del «Site Catchment Análisis» (SCA) o análisis de captación económica, dentro aún de la Nueva Arqueología, en el que se considera el yacimiento arqueológico como eje central en las relaciones hombremedio: la mejor posición es la que permite un mayor ahorro. La arqueología del yacimiento deja de ser un mero marco para pasar a ser el eje del estudio arqueológico central: definiendo el área utilizada habitualmente, rastreando los puntos de procedencia de los recursos, reconstruir los micro-ambientes y estudiar las relaciones socio-económicas (Davidson y Bailey, 1984). 


\section{Yacimientos arqueológicos y su naturaleza.}

El concepto esencial para abordar el tema del reconocimiento y análisis arqueológico del territorio es el yacimiento, por lo que cabria preguntarse en primera instancia ¿Qué es un yacimiento arqueológico?

Son varias las definiciones sobre este tema, por lo que nos permitiremos citar algunos de ellos para tener un mejor entendimiento sobre el tema a tratarse. Por ejemplo para David Clarke, un yacimiento es «...una localización geográfica que contiene un conjunto articulado de actividades humanas o sus consecuencias y a menudo un conjunto de estructuras asociadas; los yacimientos pueden ser asentamientos domésticos, centros ceremoniales, cementerios, complejos industriales o campamentos temporales» (Clarke, 1977: 11). Sin embargo esta definición entiende al yacimiento solo como un «conjunto de actividades humanas» cuando en realidad se trata de «un conjunto de vestigios o expresiones materiales de actividades humanas». Porque ya se ha establecido que la materialidad de la evidencia es lo que define más apropiadamente a la Arqueología como disciplina.

El geoarqueólogo K. Butzer, lo define como «...el registro tangible de un lugar de actividad humana en el pasado» (Butzer, 1982: 221). Enfatizando expresamente el carácter tangible o material del registro y el carácter pasado de las actividades humanas que refleja, sin embargo omite toda propuesta al carácter espacialmente delimitado de los yacimientos.

El prehistoriador E. Higgs y el geólogo C. Vita-Finzi sostienen una definición similar a la de K. Búster en la que yacimiento es «...un lugar donde existe un deposito o conjunto de depósitos que contienen evidencia de actividad humana» (Higgs y Vita-Finzi, 1972: 27). Su definición, sin embargo, no hace referencia expresa al carácter material de los vestigios, quizá porque lo hayan dado por supuesto.

Para S. y F Plog y W. Wait, un yacimiento es «una agregación de materiales culturales discreta (espacialmente delimitada, o al menos con variaciones perceptibles en la densidad de artefactos) y potencialmente interpretable (los materiales tienen suficiente entidad como para ser interpretados en términos del comportamiento humano)» (Plog y otros, 1978: 389). Insisten correctamente en el carácter espacialmente delimitado del yacimiento y en la materialidad de los depósitos teniendo en cuenta la importancia de otras localizaciones arqueológicas noyacimientos (non-sites), que son interpretables pero no discretas.

Tomando en cuenta estas y otras definiciones así como la noción de la disciplina arqueológica, la definición de yacimiento arqueológico seguida y sugerida en este trabajo seria la de una agrupación espacialmente definida y funcionalmente significativa de vestigios materiales de actividades humanas desarrolladas en el pasado en el área geográfica de Madre de Dios. 
Los limites de los yacimientos arqueológicos en esta zona muchas veces son difíciles de establecer, a causa de la acumulación de sucesivas transformaciones como por ejemplo la prolongada e intensiva presencia humana que puede generar verdaderos palimpsestos de aterrazamientos para cultivos, canales de irrigación, modificaciones de los cursos de los ríos por acción de la mano del hombre o de la naturaleza misma, grandes movimientos de tierras para trabajos de explotación minera y cantería, ejemplo: (yacimiento lítico de Huanganal), apertura, ampliación y construcción de vías de comunicación como la carretera InterOceánica Perú-Brasil (Yacimientos de Pampa Hermosa y el Triunfo), parcelación de tierras, construcción de asentamientos, aldeas, pueblos, ciudades (Yacimiento de El Triunfo), deforestaciones masivas etc., que hacen que la presencia de vestigios materiales de la actividad humana sea continua.

Otra de las exigencias para la definición de yacimiento arqueológico, es la que se refiere a su carácter funcionalmente significativo en relación con la forma de vida de la sociedad que produjo esa acumulación de residuos

Los residuos en los yacimientos arqueológicos recientemente identificados en este área geográfica, están compuestos básicamente de fragmentos de cerámica que se relacionan en un mayor porcentaje a vasijas abiertas, que morfológicamente corresponde a platos, cuencos, tazones y escudillas, con acabados finos y llanos, manos de moler, hachas de piedra en forma de «T» otras de metal, material lítico de tamaño y formas variadas, montículos de tierra oscura distribuidos de manera ordenada lo cual nos haría presumir la existencia de estructuras.

En consecuencia estos yacimientos, así como los artefactos identificados, sustentan la idea de que se realizaron actividades de procesamiento y consumo de alimentos, que culminó con la formación de residuos que en algunos casos incluyen montículos de tierra oscura distribuidos de manera ordenada que sugerirían instalaciones de infraestructura, asociadas a vasijas de consumo y una probable variedad de sobras de consumo humano.

Si bien son ciertos, estos yacimientos están asociados a restos coligados al consumo, los elementos mas notorios deben ser los montículos de tierra oscura como el del yacimiento arqueológico de Santa Maria.

Los yacimientos identificados tienen una marcada diferencia, que se nota en su dimensión, establecimiento, constitución y asociaciones que a la postre son significativos, sin embargo también hay que resaltar sus coincidencias que nos van a orientar y hacer entender su naturaleza y relación para con su medio ambiente geográfico, puesto que están asentadas entorno al río Madre de Dios, fuente inagotable de recursos. 
Estos restos aparecen primordialmente asociados al consumo y por lo tanto los elementos asociados deben de haber sido las infraestructuras utilizadas y habilitados en espacios abiertos.

\section{Yacimientos Arqueológicos investigados}

\section{a) Yacimiento arqueológico El Triunfo}

Se ubica en la margen izquierda del río Madre de Dios, frente a la ciudad de Tambopata, dentro de las coordenadas UTM WGS 84: Este 481507 y Norte 8608664 y declarado como Patrimonio a través de la Resolución Directoral No 144/INC-2006., sin una delimitación física. Recibe el nombre por estar ubicado dentro del poblado menor de El Triunfo, (Zona conocida como fundo «Rimac» de don Ernesto L. Rivero, revista Amarumayo 1972, Pág.5.), el terreno corresponde a un llano amazónico, con presencia de viviendas de un nivel y barracas de madera, que forman parte del asentamiento humano el Triunfo, los mismos se alinean en forma paralela a la carretera, en otros espacios las viviendas son dispersas, por la parte media pasa la carretera InterOceánica Perú-Brasil.

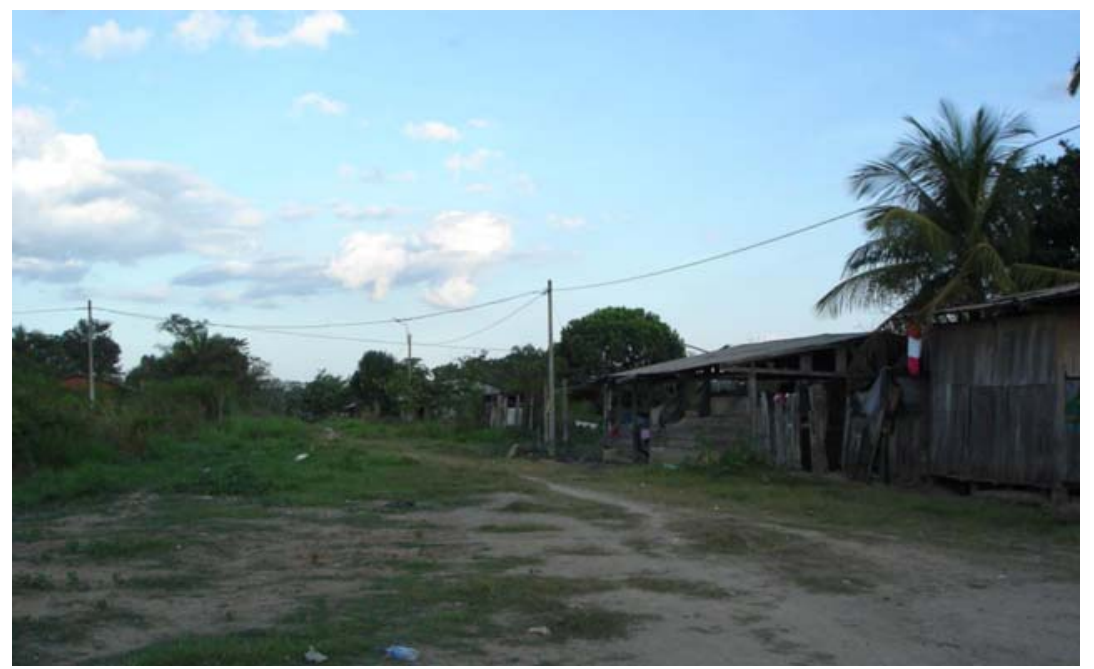

Foto 1: Y

acimiento arqueológico El Triunfo

A nivel de superficie se aprecia cerámica fragmentada de la época prehispánica, al parecer focalizados en ciertos espacios, en algunos cortes realizados al construir la carretera se puede observar claramente que la potencia cultural de ocupación fluctúa entre los 0.20 a 0.30 mts, siendo difícil definir su extensión por la naturaleza del sitio. 
Este yacimiento está compuesto generalmente de fragmentos de cerámica que corresponden en un mayor porcentaje a vasijas abiertas que morfológicamente corresponde a platos, cuencos, tazones y escudillas, en la que predominan las aplicaciones plásticas del corrugado, incisiones de formas geométricas variadas y pequeños agujeros. La potencia estratigráfica en la que se hallan los elementos culturales alcanza aproximadamente unos $0.30 \mathrm{mts}$ véase (Foto 02) y están zonificados en diferentes espacios. Finalmente después de la identificación por encargo de la empresa CONIRSA (Encargada de la construcción de la carretera InterOceánica Perú-Brasil) en los trabajos de Evaluación Arqueológica en todo este tramo, encarga a la Lic. Sofía Verastegui Y., realizar trabajos de Evaluación Arqueológica, con fines de delimitación arqueológica y un adecuado tratamiento del yacimiento.
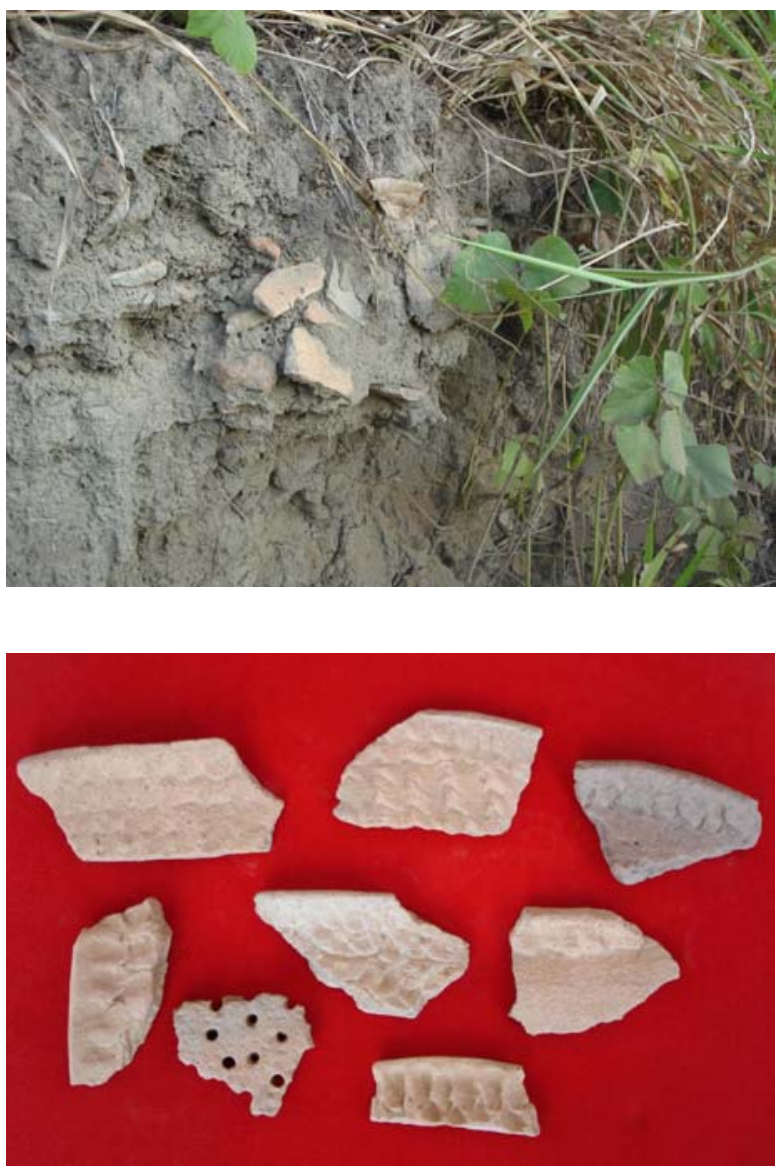

Foto 2.

Estrato cultural con presencia de fragmentos de cerámica

Fotos 3-4-5-6

Fragmentos de cerámica de EI Triunfo 


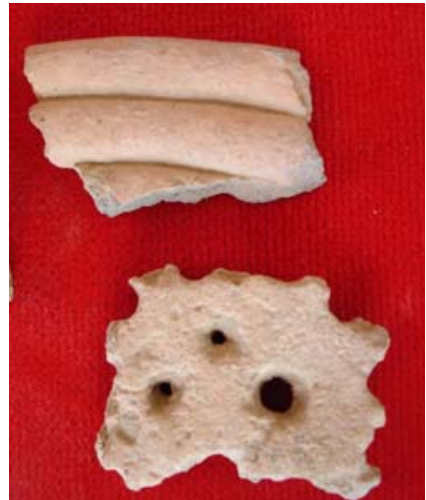

Foto 4.

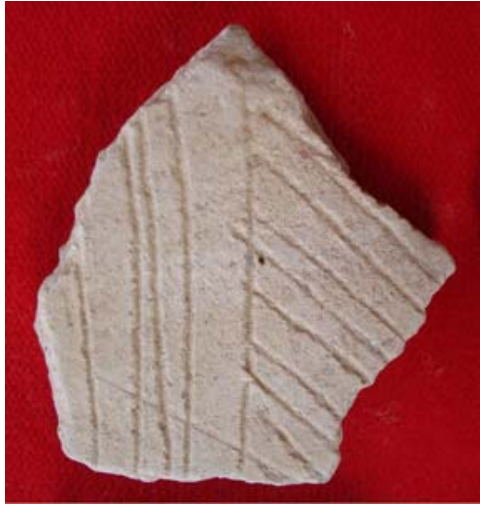

Foto 5.

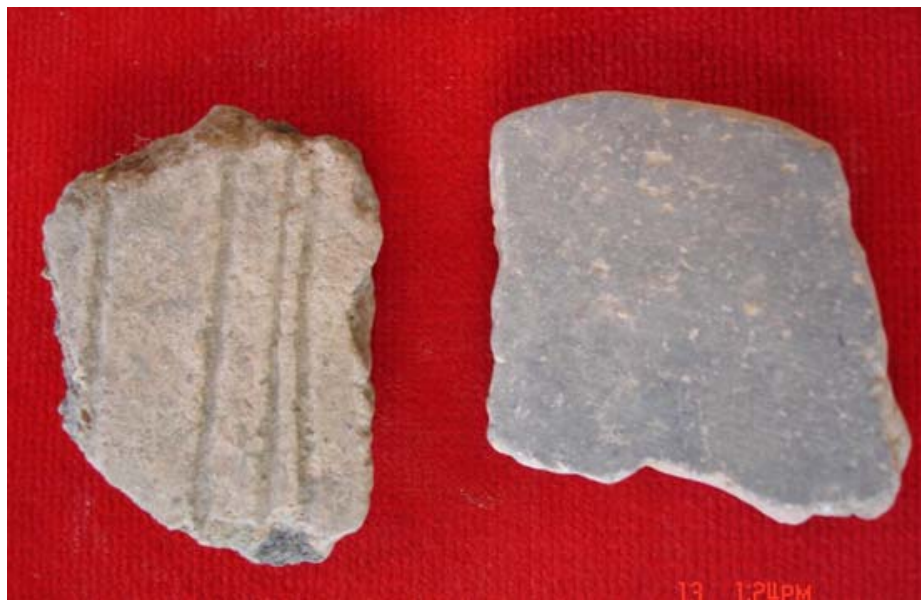

Foto 6.

\section{b) Yacimiento arqueológico de Pampa Hermosa}

Ubicado en el distrito Las Piedras, provincia de Tambopata, a la altura del Km. 50 de la carretera InterOceánica Perú-Brasil en el tramo Puerto MaldonadoIberia-Iñapari, emplazado en las Coordenadas UTM WGS 84 Este 487576,8 y Norte 8652870,38 en la margen izquierda del rió Pampa Hermosa.

Ésta se halla sobre una plataforma o terraza natural regularmente elevada de unos 10 metros de altura, cuya cima es una planicie ligeramente inclinada, con desniveles sobre una quebrada. 


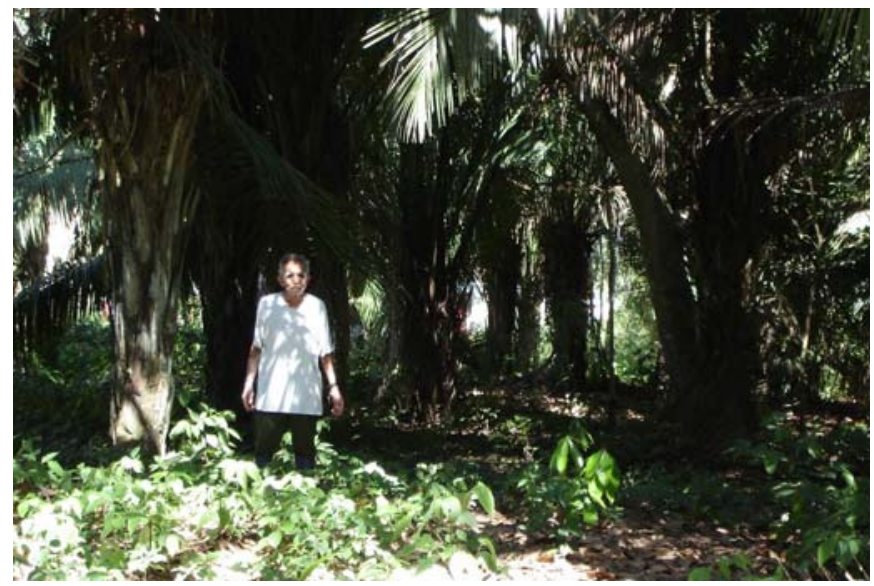

\section{Foto 7.}

Yacimiento arqueológico de Pampa Hermosa

Este sitio se encuentra actualmente dividido en dos secciones por la actual carretera en construcción, en cuya superficie se registra sectores erosionados en las que aparece el material cultural (fragmentos de cerámica y elementos líticos asociados a lajas de roca arenisca). El material cerámico corresponde morfológicamente a vasijas abiertas: platos, cuencos, escudillas y un piruro color negro con aplicaciones de líneas sinuosas. Las aplicaciones plásticas o decoraciones realizadas están en base a la técnica de la incisión en algunos casos estas son pasantes (Agujeros). Dentro del material recuperado del sitio se puede apreciar manos de moler y hachas.

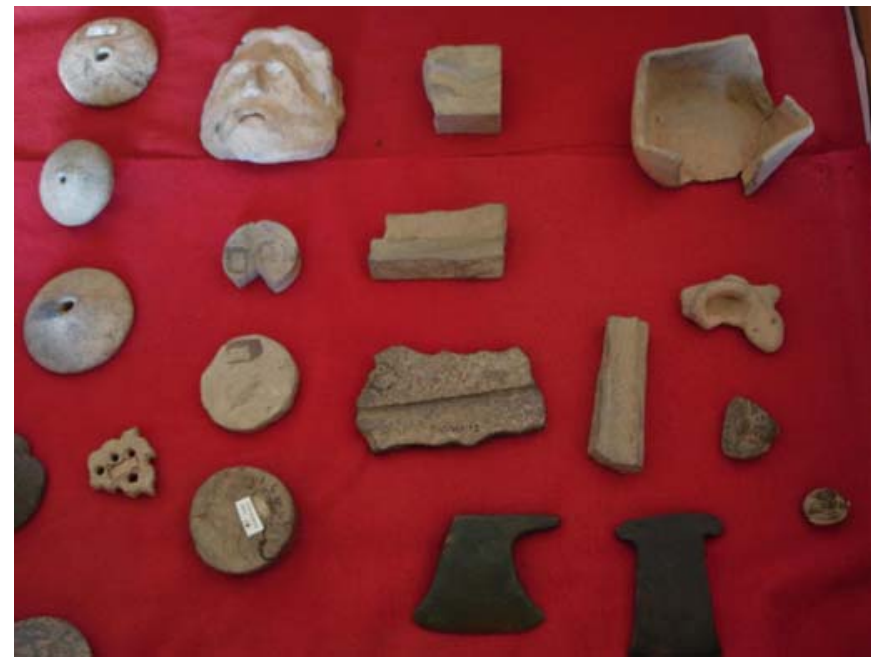

Foto 8.

Muestras de cerámica, piruros y hachas del yacimiento arqueológico de Pampa Hermosa. 
De igual modo después de la identificación por encargo de la empresa CONIRSA (Encargada de la construcción de la carretera InterOceánica Perú-Brasil) en los trabajos de Evaluación Arqueológica en el tramo, encarga a la Lic. Sofía Verastegui Y., realizar trabajos de Evaluación Arqueológica, con fines de delimitación arqueológica y un adecuado tratamiento del yacimiento.

\section{c) El yacimiento arqueológico de Sandoval ${ }^{15}$}

Se encuentra en el distrito y provincia de Tambopata, a orillas del Lago Sandoval (su origen se debe al estrangulamiento del meandro del río Madre de Dios). Dentro de las coordenadas UTM WGS 84. Este 494826 y Norte 8606854.

Desde 1974 se tiene conocimiento del emplazamiento de este yacimiento, en esta área realizaron trabajos de investigación la Dra. Concepción Gonzáles del Río entre los años 1971 y 1973, y la Dra. Inés Del Águila en el año 1974.16

La cerámica corresponde a vasijas abiertas con decoración incisa, y en menor numero a vasijas decoradas con aplicaciones de pastillaje y otras con decoración pintada de rojo sobre blanco, asociadas a tabletas (R. Ravines;1984;139), también se encontraron hachas de piedra y metal.

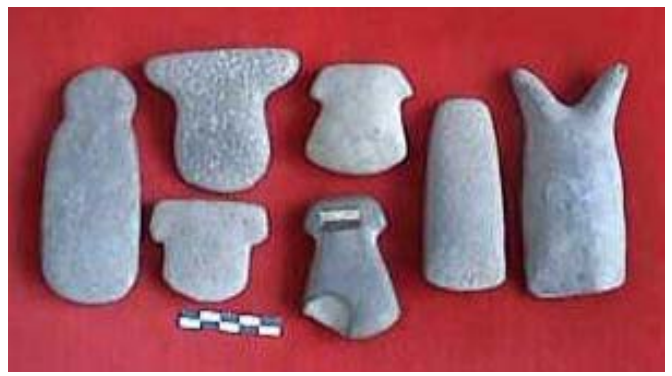

Foto 9.

Elementos líticos hallados en inmediaciones del yacimiento arqueológico de Sandoval

\section{d) Yacimiento lítico fe Huanganal}

El sitio se emplaza en el ex-denuncio minero metálico aurífero «Livia», en la jurisdicción de la Comunidad de Santa Rosa, en el distrito de Inambari, provincia de Tambopata, actualmente zona reservada como Tambopata Candamo.

Actualmente sobre este terreno se instalo el Albergue o Tambo Lodge Mejia, a orillas del respectivo lago y presenta alta erosión en superficie y los fragmentos de cerámica son muy menudos.

16 Datos tomados del Estudio de Impacto Ambiental realizados por MOBIL Exploratión, en el sector Tambopata.
} 
En las laderas de un pequeño cerro llamado como «Mira Calzón», a raíz de la denominación hecha por los mineros de la zona ${ }^{17}$, y en las cabeceras del río Jayave, en la superficie se halló material lítico de formas variadas, lo inaccesible y arcilloso del terreno mas la espesa vegetación no permitió determinar el área total y la zona de mayor concentración de estos restos, tampoco se encontró fragmentos de cerámica en superficie.

\section{e) Petroglifos Bajo Madre De Dios}

El sitio se localiza en el fundo ganadero del Sr. Gregorio Maceda Maceda, en la zona denominada Bajo Madre de Dios, en el distrito Las Piedras, provincia de Tambopata. Dentro de las coordenadas UTM WGS 84. Este 485048 y Norte 8607112.

Se trata de un bloque de roca en arenisca que está fragmentada por uno de sus lados y dispersa en un área de $5 \mathrm{~m}^{2}$., la misma que tiene representaciones de rostros estilizados y figuras geométricas, elaborados en bajorrelieve. Recuperado del cauce del río Madre de Dios de un conjunto de tres rocas fragmentadas que formaban un solo bloque.

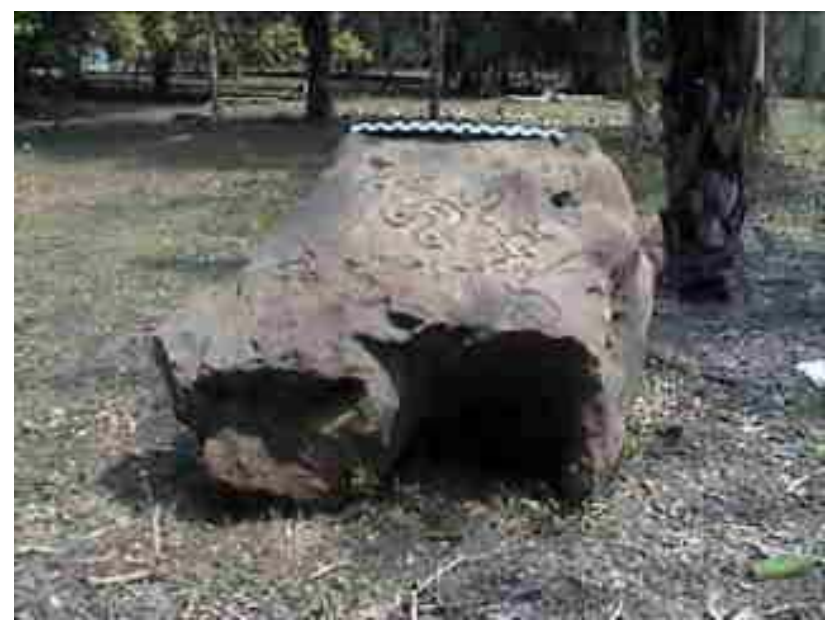

Foto 10.

Petroglifo Bajo Madre de Dios

17 Comunicación personal del Sr. Florentino Quispe Condori, quien me sirvió de guía en esta zona, de donde también recuperamos restos fósiles que corresponden a un glossoterium. 
Asociado a esta evidencia cultural en el área del centro poblado de Bajo Madre de Dios, se determin la existencia de otros dos yacimientos arqueológicos que contienen fragmentos de cerámica, distribuidos en un área de $700 \mathrm{~m} 2$, con una orientación Sureste en la margen derecha del río Madre de Dios, en los que predomina también la cerámica corrugada.

\section{f) Yacimiento de cerámica Gamitana ${ }^{18}$}

Se encuentra en el Fundo Castañero de la Familia Gonzáles Erpillo, en el distrito Las Piedras, provincia de Tambopata.

Los fragmentos de cerámica fueron ubicados en el sector denominado «Quebrada Gamitana», en el denuncio castañero del Sr. José González Erpillo, en un área aproximada de 2,000 m2. Dentro de sus características decorativas presentan muescas o corrugado en el entorno del cuello de las vasijas. En la superficie del terreno no se ubicó evidencia de estructura arquitectónica alguna.

\section{g) Yacimiento de cerámica Santa María}

Ubicado en el Fundo Santa María jurisdicción del distrito Las Piedras, provincia de Tambopata. Se encuentra sobre una terraza elevada (tahuampa), cuya cima es una planicie con ligeros desniveles. Emplazada en las coordenadas UTM WGS 84. Este 484.092 y Norte 8621660.

La presencia de material cultural se encuentra dispersa tanto en superficie como en los taludes de la terraza. La definición de algunas áreas con presencia de cerámica se debió al roce y quema que se realizaron en esta zona. En el corte del terreno se puede apreciar una primera capa de tierra con humus y raíces, una segunda capa de tierra oscura con una potencia entre 0.50 a $0.65 \mathrm{mts}$ de promedio con presencia de fragmentos de cerámica.

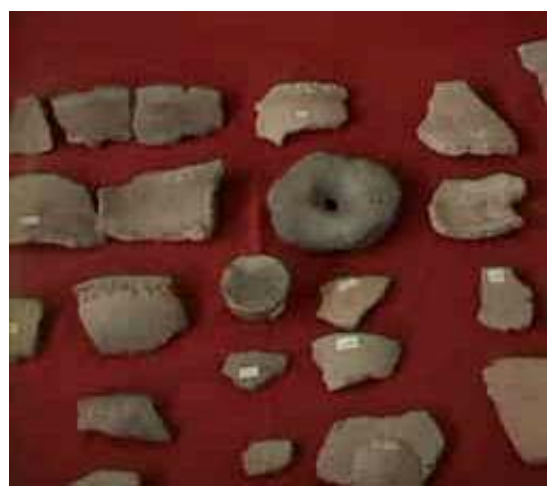

Foto 11.

Fragmentos de

Cerámica Santa María

18 Realizó pozos de prueba en 1998 el Antropólogo Florentino Champi. 
El análisis del material de superficie nos indica la presencia de vasijas abiertas: platos, cuencos y tazones. Algunos fragmentos de cerámica presentan diseños decorativos realizados con la técnica de la incisión.

Este sitio arqueológico al extremo Sur está asociado a un ojo de manante de agua, área donde presenta montículos de tierra oscura distribuidos de modo ordenado, lo cual indicaría la existencia de estructuras (viviendas)

Roger Ravines, en mayo de 1994, al visitar los trabajos de explotación aurífera en el sitio denominado Playa del Águila, a $30 \mathrm{Km}$ de Laberinto, denuncio minero del Sr. Juan Méndez, refiere; se trataba de un cementerio ubicado en la terraza del río, y no guarda mayor relación con la actual cerámica tradicional de la zona. Sus vasijas de cuerpo compuesto, en la que destacan pedestales altos, ostentan generalmente decoración geométrica pintada de color rojo sobre fondo blanco. Aunque es imposible fijar una edad a estas vasijas, su forma y decoración indican una cierta relación con la tradición Cumancaya del Ucayali Central. (R. Ravines; $1984,139)^{19}$

También de esta área el profesor Niel Medina Mugaburo ${ }^{20}$ en el año 1998, en la zona denominada Playa Los Pachecos, en el denuncio minero Paquisha, recuperó material alfarero con las características semejantes a las que indica Ravines, lamentablemente estas dos áreas arqueológicas por el trabajo desmedido en la explotación del oro, fueron completamente removidos en sus suelos. Otros sitios con información documentada que corrieran la misma suerte, fueron los ubicados por Sheila Aikman (1980) en el distrito de Madre de Dios, provincia de Manu, donde realizó algunos trabajos de prospección en la cuenca del río Karene (Colorado) llegando a identificar cinco sitios con presencia de cerámica y hachas de piedra.

Estos sitios a los que hace referencia son: San Josée ${ }^{1}$ se halló fragmentos de cerámica llana y fina; Puerto Luz, hachas de piedra en forma de T: Palizada, fragmentos de cerámica llana y fina; Chapajal A, fragmentos de cerámica llana y fina correspondientes a cuencos y ollas y Chapajal B, algunos fragmentos de cerámica fina no identificada.

Durante la época inca, no se ha documentado ocupación en territorio de Madre de Dios. Aunque podemos referir que había algún nivel de intercambio comercial puesto que se pueden registrar algunas evidencias arquitectónicas de la presencia Inca, los cuales están identificados en la zona de Manu: Mameria, El mirador ${ }^{22}$, Llaqtapampa y la vía troncal del Antisuyu asociado a un camino secundario entre

\footnotetext{
19 Parte de lo que quedaron de estos objetos de cerámica fueron cedidos por el Municipio provincial de Tambopata al INC-MDD, para su conservación.

Trabajador del INC-MDD, parte de este material también se encuentra en esta sede del INC. Cabe indicar que estos territorios actualmente están ocupados por los Arak'mbut, (Reserva Comunal Amarakaeri) quienes también participaron en la ubicación de estos sitios. 
Shintuya y las cabeceras del río Azul en la que se registró la existencia de un trazo del Qhapaq Nan que se introducía hasta esos confines. En el siglo XIV el límite territorial entre los Incas y sus contemporáneos de esta región, eran las minas del Alto Beni; Tipuani, Mapiri y Chipullisani, zonas actualmente en territorio Boliviano al Sur Este del Parque Nacional Bahuaja - Sonene (Saignes 1981). Sin embargo, hay indicios de un camino incaico con varios caminos secundarios en el tramo que va desde Ayaviri hacia Macusani y Ollaechea en la cuenca del Río Inambari, al Sur del Tambopata (Von Hagen 1955; Cieza de León 1984).

En la ubicación de estos sitios arqueológicos, debemos precisar que los trabajos arqueológicos realizados hasta hoy son de identificación restringida y solo permiten aproximaciones en algunos de estos sitios, siendo necesario a futuro corroborar mediante trabajos de prospecciones extensivas, de tal modo que permitan plantear definiciones cronológicas y caracterización cultural; procedencia y relaciones de intercambio.

Podemos aproximarnos hacia una cronológica relativa en virtud a que la cerámica recurrente de los yacimientos arqueológicos identificados para las zonas comprendidas entre El Triunfo (Puerto Maldonado) hasta la frontera con Bolivia (Lago Valencia) emplazadas en ambas márgenes del río Madre de Dios, por la morfología que presentan los restos de ceramica, debido a que la decoración mas predominante es la corrugada en los sitios de El Triunfo, Bajo Madre de Dios, Gamitana, Lago Sandoval, Briolo y Lago Valencia. ${ }^{23}$

En trabajos realizados por Parssinen y Siirianianen ${ }^{24}$ refieren que la alfarería corrugada parece haber estado presente en la costa sur de Brasil, entre las tradiciones Taguara y Una, que este estilo cerámico se difundió junto a la expansión Guaraní, probablemente desde el Amazonas central hasta el Madeira o Guapore, prosiguiendo luego al centro del río Paraguay, entre los años 1 y 500 d.C. el estilo parece haber aparecido también en el Medio Ucayali, en los alrededores del año 700 d.C. (Lathrap et al. 1987:225-71; Myers 1988:64,7; Silva Noelli 1998:655.656; ver también Meggers y Evans 1983:317).

L. Medina, R. Marín, V. Chamorro ${ }^{25}$ al referirse sobre el tema cronológico de la ocupación con decoración corrugada, indica su similaridad a las muestras de los

23 De Briolo, Lago Valencia y Km 7 carretera a Iberia de Puerto Maldonado, se tiene solo muestras de fragmentos de cerámica, esperamos ubicarlos en el lapso de nuestra permanencia en Madre de Dios.

$24 \quad$ Parssinen Martti \& Ari Siiriainen «¿Cuándo empezó, realmente, la expansión Guaraní hacia las vertientes andinas orientales?» En: Andes Orientales y Amazonía Occidental, ensayos entre la historia y la arqueología de Bolivia, Brasil y Perú. Colección Maestría en Historias Andinas y Amazónicas. Universidad Mayor de San Andrés. Vol. 3: 215.232. Producciones CIMA, La Paz, 2003.

25 L. Medina, R. Marín, V. Chamorro «Investigaciones Arqueológicas en la zona del Bajo Urubamba» En: Estudios Amazónicos, Abriendo Fronteras Nº 1 Agosto-Diciembre 2004: 17-27 Centro Cultural «Jose Pío Aza» Misioneros Dominicos, Lima-Perú. 
sitios de Camisea 1 con $632( \pm 160)$ hasta los 1003 años d.C. Manirorato con 850 años d.C y Camisea 2 con 1003 d.C. para la zona del Bajo Urubamba. En las vertientes andinas en el oriente Boliviano fue datado alrededor de 1,500 d.C. mediante la analogía de registros etnohistóricos.

Pese a que la investigación arqueológica en esta región está en sus fases iniciales, siendo aun no sistemáticas, quedan evidenciados asentamientos altamente organizados, adaptados a diferentes situaciones ecológicas, dentro del contexto cultural amazónico del precontacto, sin embargo esperamos más adelante ya referirnos a la dimensión de los sitios y los parámetros de tamaño poblacional y aproximarnos a la distribución o tipología de las estructuras y las características de cada sitio para finalmente hacer referencia a un orden cronológico de los mismos.

\section{Comentarios Finales}

La cerámica de estos yacimientos recientemente identificados en sus aspectos generales, se caracteriza por la utilización de desgrasante de tiesto molido; representados en grandes platos discoidales, levemente cóncavos, con estrías paralelas y cruzadas (ralladores); manos fusiformes de arcilla con estrías o decoración puntiforme incisa; trozos de barro batido, tal vez de paredes; soportes para apoyar piezas sobre el fuego; la decoración que predomina es incisa (muy fina, fina y mediana), con escasa representación de aplicaciones y adornos así como la baja frecuencia de cerámica pintada, las mismas que están en su mayor porcentaje en proceso de deterioro y erosión de sus superficies, por otro lado los hallazgos de hachas de piedra con garganta y con aleta, manos de moler, por la naturaleza de los suelos son escasos.

Los motivos decorativos que hemos podido identificar son el de la Decoración Corrugada.- Definidas como impresiones digitales y ungulares (de uñas), hechas intencionalmente por el alfarero, en vasijas en «cuero»; que denotan una superficie áspera e irregular que permite el manipuleo y uso artefactual de los objetos hechos en cerámica, los que se ha caracterizado en:

- Impresiones digitales de forma redondeada, oblicua o vertical, en series de dos a más filas secuenciales. En el borde y carena del cuerpo de vasijas abiertas de la forma 1 y 4 . Y en el borde y/o parte superior e inferior del cuerpo de vasijas cerradas de las formas 4 y 6 .

- Impresiones digitales en una fila, intercalados a depresiones horizontales producidos por la superposición de capas de arcillas.

- Decoración Incisa-corrugada. 
Decoración mixta, que se asocia a vasijas cerradas, definida como impresiones digitales oblicuos, en uno o dos filas, por debajo de cada depresión horizontal, asociados a decoración lineal incisa.

En suma, la poca información, primordialmente incompleta, en relación con la gran extensión territorial, plantea que estamos en presencia de un espacio que aún necesita imperiosamente de la ejecución de investigaciones sistemáticas antropológicas y arqueológicas en sus diferentes especialidades. En los sectores adyacentes a la carretera transoceánica se desarrollaron sucesiones preliminares que se pueden verificar, basadas principalmente en complejos cerámicos y formas de disposición. No obstante la destacada cantidad de información dada a conocer en los últimos años, en especial en estos sectores que indicamos, el resto del gran territorio plantea una incógnita que de no ser investigada desaparecerán las condiciones de su estudio e interpretacion en función de la rápida transformación ecológica. Resulta preciso y urgente abocarse a la problemática, en nuestro caso arqueológico, porque es imperioso saber con exactitud que papel le toco a este sector geográfico en el desarrollo y/o dispersión de rasgos culturales.

\section{Bibliografía}

\section{AIKMAN, Sheila.}

1983 «Informe preliminar sobre hallazgos arqueológicos del río Karene (río colorado), Madre de Dios». En: Amazonía Peruana. Centro Amazónico de Antropología y Aplicación Práctica. Vol. IV Nº 8: 93-101 Lima Perú.

1985 «Informe preliminar sobre hallazgos arqueológicos del río Karene (río colorado), Madre de Dios» En: Amazonía. Cerámica y rutas de Intercambio. Pp. 77-85. Iquitos. Dirección Departamental del Ministerio de Industria, Turismo e Integración 


\section{BOLÍVAR, Y. Wilber.}

2006 «Informe $N^{o} 083$ - WBY-DRC-C-DCPCI-SDCH-2006» Sobre trabajos para delimitacion de sitios arqueológicos de El Triunfo y Pampa Hermosa en al Provincia de Tambopata- Maldonado- Madre de Dios. Presentado a la Dirección Regional del INC-Cusco.

\section{BUTZER, K.}

1989 «Arqueologia». Una Ecologia del Hombre, Barcelona, Belleterra. $1^{\text {a }}$ edicion inglesa 1982

CLARKE, D.L.

1977 «Spatial information in archaeology». En: Clarke, D.L. Ed. Spatial Archaeology, 1-32. londres, Academic Pres

\section{GARCÍA SANJUÁN, Leonardo}

2005 Introducción al reconocimiento y análisis arqueológico del territorio. $1^{a}$ edición, Editorial Ariel, S. A. Barcelona, Cáp. 2, pp. 23-25. España

\section{HIGGS. E. S., VITA-FINZI, C.}

1972 «Prehistoric economies: a territorial approach». En: Higos, E.S. (ed). Paper in Economic Prehistory. Pp. 27-36 Cambridge University Press.

\section{KLARICH, Elizabeth. A.}

2008 «QQuienes eran los invitados? Cambios temporales y funcionales de los espacios públicos de Pukara como reflejo del cambio de las estrategias de liderazgo durante el periodo formativo tardío. Perspectivas teóricas acerca de los espacios públicos en Pukara». En: Encuentros: Identidad, Poder y Manejo de Espacios Públicos. Publicación del Boletín de Arqueología PUCP 9, 192-197, Lima.

PLOG, S.; PLOG, F.; WAIT, W.

1978 «Decisión making in modern surveys». En: Schiffer, M.B. (Ed.), Advances En Archaeological Method and theory, 1, pp. 384-421. Nueva Cork, Academia Press.

\section{RAVINES, Roger.}

s.f. Historia del Perú. Primero de Secundaria. Editorial Brasa S.A.

1993 «Cerámica Arqueológica del rió Tambopata, Madre de Dios». En: Boletín de Lima, Vol. XV, No 90, pp. 15-20. Lima. Editorial los Pinos.

\section{MAQQUE, A. Rubén.}

2000 «Madre de Dios y un poco de su pasado» En: Diario Mi Frontera. Año 9. № 51 Puerto Maldonado, Julio-2000 


\section{MARTTI, Parssinen, SIIRIAINEN, Ari.}

2003 «Cuándo empezó, realmente, la expansión Guaraní hacia las vertientes andinas orientales?». En: Andes Orientales y Amazonía Occidental, ensayos entre la historia y la arqueología de Bolivia, Brasil y Perú. Colección Maestría en Historias Andinas y Amazónicas. Universidad Mayor de San Andrés. Vol. 3: 215-232. Producciones CIMA, La Paz, 2003.

\section{MEDINA, L., MARÍN, R. y CHAMORRO, V.}

2004 «Investigaciones Arqueológicas en la zona del Bajo Urubamba». En: Estudios Amazónicos, $\mathrm{N}^{\circ} 1$ Agosto-Diciembre 2004: 17-27 Centro Cultural «José Pío Aza» Misioneros Dominicos, Lima-Perú.

MEGGERS, B.J.; EVANS, C.

1978. «Lowland South America and the Antilles». In: Jennings, J.D. (Ed.), Ancient Native Americans, Chapter 12:543-591. San Francisco. Freeman.

\section{MEGGERS, B.J.}

1997 «La Amazonía en vísperas del contacto europeo: perspectives etnohistóricas, ecológicas y antropológicas». In: Varón Gabai, R.; Flores Espinoza, J. (Eds.) Arqueología, Antropología e Historia de los Andes. Homenaje a María Rostworowski, p. 93-113. Instituto de Estudios peruanos. Lima, Perú.

\section{MOBIL EXPLORATION AND PRODUCING PERU INC}

1994 Estudio de Impacto Ambiental (EIA) Prospección Sísmica, Sector Tambopata. Informe mayo 1994.

\section{PAREDES PANDO, Oscar.}

1996 Amazonía: 500 Años. Editorial de la UNSAAC-Cusco.

\section{VEGA-CENTENO, A. Patricia M.}

2003 «Algunos apuntes sobre la investigación etno-arqueológica de un sitio con petroglifos en la selva sur peruana». En: Arqueológicas 26 del Museo Nacional de Arqueología Antropología e historia del Perú. Publicación del Instituto de Investigaciones Antropológicas Instituto Nacional de Cultura. Lima/2003. Vol.26:59-71.

\section{VEGA-CENTENO, Rafael.}

2008 «Consumo ritual en la construcción de espacios públicos para el Periodo Arcaico Tardío: el caso de Cerro Lampay. La naturaleza de los depósitos de basura». En: Encuentros: Identidad, Poder y Manejo de Espacios Públicos. Publicación del Boletín de Arqueología PUCP 9, 107-111, Lima. 